\title{
Radio over Fiber networks synthesis using meta-heuristic BCO
}

\author{
Alexander Pereverzev ${ }^{1,2, *}$, Dmitry Ageyev ${ }^{1,2}$ \\ ${ }^{1}$ Telecomunication system, Kharkiv, Ukraine \\ ${ }^{2}$ Kharkiv National University Radio Electronics, KNURE, Kharkov, Ukraine
}

Email address:

pereverzev_aa@mail.ru (A. Pereverzev),dm@ageyev.in.ua (D. Ageyev)

\section{To cite this article:}

Alexander Pereverzev, Dmitry Ageyev. Radio over Fiber Networks Synthesis Using Meta-Heuristic BCO, Communications. Vol. 1, No. 1, 2013, pp. 9-15. doi: 10.11648/j.com.20130101.12

\begin{abstract}
Radio access network over fiber (RoF) technology combines both wireless and fiber-optic technology. The advantages of this technology are low attenuation, bigger network bandwidth, easy installation and management, reduced power consumption, multiservice, dynamic allocation of resources. There are many methods of designing optical and wireless networks. Since RoF combines these technologies, the design tasks are a complex problem. Therefore there is a need for a new design method for RoF technology. The main problem in designing DWDM transport networks is the wavelength assignment of light paths. If the network topology has a great need to use heuristic methods there is one way to solve this problem: use of the BCO-RWA algorithm. Meta-based heuristic algorithm BCO-RWA contains the modeling of the behavior of bees collecting nectar. The main advantage of this algorithm is low computational complexity. However, BCO-RWA has the following disadvantage: during the calculation of route selection probability, base algorithm does not take into account the nonlinear four-wave mixing phenomenon. In this paper we present new method to design RoF network and modification of BCO-RWA algorithm.
\end{abstract}

Keywords: RoF, BCO-RWA, Route, Light Path, Design, Connection, Nonlinear Four-Wave Mixing Phenomenon DWDM

\section{Introduction}

A promising method in networks building is the use of two technologies - wireless and optical. The advantage of the use of such networks is that the wireless technology provides the frequency for signals between base stations. it is recommended due to mobility, easy use and low cost and avoids limitations connected with bandwidth of optical technologies which are used in transport network. For example networks, which consist of the integration of wireless and fiber networks, known as Radio-over-Fiber. Radio-over-Fiber (RoF) technology combines wireless and fiber-optic technology to improve the efficiency of use of these technologies. In [1,2] RoF network using the optical section provides reliable transmission of a radio signal with a small attenuation in the fiber, while the wireless part provides mobility at low cost. In these networks, radio frequency (RF) signals are transmitted in optical fibers.

These networks are connected through a base station controllers (BSC) and remote antenna (RA), which are connected via fiber channel.
The functionality of the technology is performed by the BSC controller.

RA provides only converting of RF signals. All radio resources are identified by base stations (BS), which are in the BSC and these resources can be dynamically allocated across the network using a variety of configurations. The main advantages of RoF technology include low attenuation, more network bandwidth, ease of installation and management; reduce the power consumption, multiservice, dynamic allocation of resources.

In [1] the problem of efficient use of radio resources in the access network RoF is shown. The problem of dynamic resource allocation is important. For example of GMS traffic in a RoF distribution system: more capacity can be allocated to a certain area during the peak times and then reallocated to other areas when off-peak. [3].

The model provides an effective partitioning or merging cells during the traffic consumption of mobile subscribers.

In [4] the problem of maximizing distribution traffic via the ROF, which is achieved in the efficient allocation of radio antennas with the influence of interference is solved. This task is reduced to an integer linear programming, but 
this approach is used only for a fixed number of users as well as for dynamically changing customer uses heuristic methods to solve this problem.

In this paper the RoF integrated with optical DWDM technology. DWDM technology provides the most extensive and cost-effective way to expand the bandwidth of fiber-optic channels to hundreds. DWDM enables the efficient exploitation of the fiber network's bandwidth.

There are exist many methods for the design of optical transport networks $[5,6]$ and wireless access networks $[7,8,9]$. However, for designing RoF technology that provides the base platform for the integration of wireless and optical network system. There is a need to develop new method of design.

But solving problems in the design of wireless and optical technologies at the same time is difficult. In this paper we propose new method of design RoF networks that involves principles of decomposition.

\section{Design of RoF}

\subsection{New Method for Design of RoF}

Main tasks in the design of RoF access network are following:

- planning of spatial deployment for antennas;

This task means the synthesis of wireless access network, which includes the distribution of antennas with certain direction and appointment of frequency channels taking into account subscriber density, antenna coverage, signal strength and interference noise;

- synthesis of the network topology;

This task can be viewed in two ways: during construction of a new network or use an existing network. The first variant implies a synthesis of network topology with a connection of at least three nodes, finding all the shortest routes in the network provided the uniformity of flow distribution over the network. In the second variant we use additional distribution of the nodes in the network and search of all the shortest routes according to the above-mentioned criteria.

- The choice of routes and of distribution for route wavelength assignments in the optical network (RWA). This problem determines the choice of routes and route wavelength assignments according to the criterion of optimality.

The proposed method consists of the decomposition approach, in other words the main tasks in the design are solved separately in a specific order, the data obtained as a result of their solution considered to be the initial data for the solution of other problems. Fig. 1 shows the block diagram of the algorithm for solving the problem of RoF network design.

Design method access network Radio over Fiber

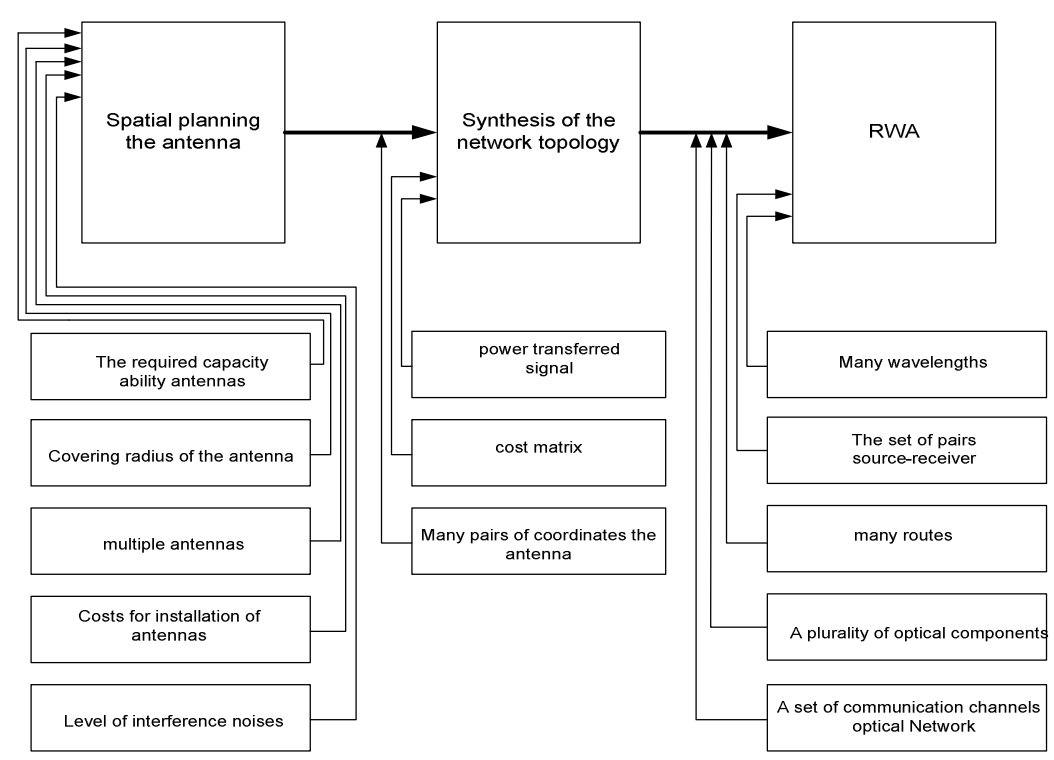

Figure 1. The block diagram of the algorithm for solving the problem of network design based on RoF technology.

Therefore, the algorithm assumes a strict sequence of subtasks solutions, which are shown below:

1. Solution for the territorial planning of antennas, then, this solution will be the initial data for the solution of the synthesis for the network topology.

2. Problem of topology synthesis. The found solution is the input for the task route selection and appointment of the routes of wavelength distributions in the optical network (RWA).
3 Solution of the choice of routes and distribution of route wavelength assignments in the optical network (RWA).

\subsection{Method of Planning $R A U$}

Standard model for solving the problem for planning of antennas, reduced to a wireless network with a set of transmitting and receiving antennas, which should be 
placed on the territory. Each antenna is characterized by the position (geographic coordinates and altitude) and a range of electronic options. The process of network design means determination of location and purpose of the frequency antennas. As a result, the network is estimated using the two main factors: the connection to the network and the quality of signal transmission bandwidth.

In general, when placing antennas needed to meet the basic criteria - coverage and capacity of the antenna. The template is used to format your paper and style the text. All margins, column widths, line spaces and text fonts are prescribed; please do not alter them. You may note peculiarities. For example, the head margin in this template makes measurements more proportionately than customary.

In this paper we propose a method for the solution for the problem of territorial planning of antennas [10].

\subsection{Method of Synthesis of Network Topology}

In the design of telecommunication system it is necessary to determine the parameters of the structural elements of the system and the synthesis of its structure (topology). Synthesis of the network topology is one of the main problems in the design of any hierarchical multiservice network. This issue is devoted to a large number of works [11, 12]. For solving problem of topology synthesis method of M-structures is used.

In the method of M-structures the initial structure of a given connection is not chosen by chance, but it is deliberately generated using a special algorithm. Then initial structure M0 is minimized by a minimum value when all of the restrictions introduced.

After synthesis of the network topology it is necessary to search for the set of shortest routes, but the choice of route should take into account the condition of uniformity of communication channels that avoided a large number of bottlenecks in the network.

For solving problem of the search for the set of shortest routes use Yen's algorithm is proposed of [13].

\subsection{Method for Solving of RWA Problem}

Optic network connects the antenna and the host controller in the network and is used as RoF transport network to transmit radio signals over fiber. Optical network technology is built by DWDM technology. When using a network based on DWDM technology the main tasks of design are: routing and destination wavelength light paths (RWA).

To solve the problem of the distribution of wavelengths in optical networks (RWA) a variety of methods are used. Some of them use traditional optimization techniques that will really find the global optimum, such as integer linear programming, discussed in $[16,19]$.

This approach to the problem faces the problem of "combinatorial explosion" (the sharp increase in the number of restrictions with the increase of the number of nodes and streams in the network), which increases the computational complexity of the problem.
This problem can be solved due to the use of heuristic algorithms, such as Tabu-search [18, 19], the annealing algorithm [20,21], or genetic algorithms [22].

The solution of the wavelength distribution of the light paths is considered in [22] by using a genetic algorithm, which is based on modeling of the evolution and use of operations such as inheritance, mutation, selection and crossover. However, this method has the disadvantage: the algorithm finds the area of a local extreme of the objective function, and then a large number of iterations is spent on improving local extreme point of clarification, instead of finding the global optimum, which leads to an increase of time in solutions of the optimization problem.

At the heart of the annealing algorithm [18] is the use of random search orderly based simulation of the formation of the crystalline structure in the material with the lowest energy in cooling.

The advantage of the annealing algorithm is avoiding "traps" in the local extreme of the function by taking not only the changes in the parameters that lead to a decrease in value of the function, but also some changes that increase its value, depending on the temperature parameter characterizing modulated process.

However, the disadvantage of this method is the high computational complexity; this happens due to the fact that good solution requires a very slow decrease in the probability of transition for state with a higher value of the objective function, which leads to a large number of iterations of the algorithm.

Tabu-search is a meta-heuristic local search algorithm, which uses a local search procedure that consists of repeated transition from one possible solution to the improvement of other solutions, but forbidding those movements that return search to the previous solution and lead to an infinite loop of the algorithm. The basic principle, which prevents the algorithm from falling into local optimum is a taboo list, that is updated at the end of each iteration.

At each iteration, the algorithm improves the current solution found with the previous solutions. It uses an array of previously found solutions. One major problem of taboo search algorithm is that the algorithm can be effective only in discrete spaces, the introduction of penalties for violation of the restrictions in the objective function does not guarantee feasible solutions.

The basis of meta-based heuristic algorithm BCO-RWA $[21,12]$ is the modeling of the behavior of bees collecting nectar. The main advantage of this algorithm is low computational complexity. The method of bee colonies is better than others, it demonstrates the ability to come out of local optima by the use of random search.

As the number of vertices of an advantage to the bee algorithm listed above algorithms in terms of quality of the solution increases. Therefore, in this paper, we propose to use the Meta-heuristic BCO-RWA for allocation of wavelengths.

The method of bee colonies is better than others, that demonstrate the ability to come out of local optima by the 
use of random search. As the number of vertices, the advantage of bee algorithm in comparison to listed above algorithms increases in terms of solution quality.

Therefore, in this paper we propose use of the meta-heuristic BCO-RWA for allocation of wavelengths.

At the beginning of the search process all the bees are in the hive. Bees fly from the hive and fly around all the flowers. Flight of bees is divided into stages. At each stage of the algorithm a bee chooses to visit one flower at each stage. This process corresponds to the connection between the source and destination pairs - total length of the wave along the optical channels in the selected route between source and destination.

During the phase of solution search each bee visits $n$ stages (search of $\mathrm{n}$ new light paths). At each stage a bee chooses one of unvisited flowers. $\mathrm{N}$ sequentially visited by a bee flowers display a particular solution of the optimization function.

The bee's attendance of $\mathrm{n}$ steps is equivalent to setting new connections between a pair of source and destination of the light path through the successful installation of light path that depends on the number of available wavelengths in the optical channels through which the route is made. After the phase of finding a solution, the algorithm proceeds to the decision selection.

Probability $\mathrm{p}$ of that a bee will choice unvisited flower can be described by the expression:

$$
p=\frac{1}{n_{t}}
$$

where $n_{t}$ is the total number unvisited nodes.

When selecting the next flower algorithm choices the route among many available ones between a pair of source-recipient and destination of wavelengths along the selected route.

The choice of the physical route in the optical network in BCO-RWA algorithm is random, which is described according to the logical model [19] and is as follows:

$$
p_{r}^{s, d}=\left\{\begin{array}{l}
\frac{e^{V_{r}^{s, d}}}{\left|R^{s, d}\right|}, \forall r \in R^{s, d}, W_{r}>0 \\
\sum_{i=1} e^{V_{i}^{s, d}} \\
0, \forall r \in R^{s, d}, W_{r}=0
\end{array}\right\}
$$

where $\left|R^{s, d}\right|$ - total number of available routes between

the pair. The route is available if there is at least one unoccupied wavelength along the route. The weighting factor, which is used in (1) with the choice of the physical route is calculated by the following expression:

$$
V_{r}^{s, d}=\left\{a \frac{1}{h_{r}-h_{r \min }+1}+(1-a) \frac{W_{r}}{W_{\max }}\right\},
$$

where $r-$ serial number of the route between two nodes $(s, d), r=1,2, \ldots k, r \in\left\{R^{s, d}\right\} ; h_{r}$ - length of the route; $h_{\min }-$ length of the shortest route; $W_{r}-$ the number of wavelengths available on a route $r$ $r=1,2, \ldots k, r \in\left\{R^{s, d}\right\} ; W_{\max }-$ maximum number of wavelengths available on all routes; $a-$ weighting factor $0 \leq a \leq 1$.

After bees return to the hive, the algorithm decides to leave a particular solution of the optimization problem or extend a particular solution that was found in the next phase of finding a solution. In the exchange of information between the bees, each bee can get information about the particular solutions of other bees. The probability that a bee will start a solution phase $u+1$, using the same path as in the phase $u$ of finding a solution is described by the following expression:

$$
p_{b}^{u+1}=e^{-\frac{C_{\max }-C_{b}}{u}},
$$

where $C_{b}$ - the total number of lightpaths established from the beginning of the search and that match the route found $\mathrm{b}$-th bee; $C_{\max }-$ the maximum number of lightpaths established from the beginning of the algorithm.

The probability $p_{k}$ that in $k$ the exchange of information between bees (analysis of a particular solution), the found route will be chosen by a free bee is as follows:

$$
p_{k}=\frac{e^{C_{p}}}{\sum_{p=1}^{P} C_{p}}
$$

where $C_{p}-$ the total number of light paths established in $k$-the exchange of particular solutions.

However, the analysis of the algorithm identified the following shortcomings:

- it does not solve the problem of choosing the location of the optical convector in the network (in the original paper converter is installed in the first node of the route in the absence of the possibility of connection);

- The stage of the probability calculation for selecting a route does not consider convectors in nodes, through which route is made and within the algorithm they are limited to only one convector.

- The impact of four-wave mixing in the installation of light paths is not regarded.

\subsection{Modification of Method for Solving RWA}

At the stage of calculating the probability of choosing a route the expression has been modified considering sign to the condition that the node, through which the route is made, may have an optical convector. The modified expression for the probability of route selection by a bee is:

$$
V_{r}^{s, d}=\left\{a \frac{1}{h_{r}-h_{r \min }+1}+(1-a) \frac{W_{r}^{*}}{W_{\max }}\right\},(6)
$$

where $r-$ serial number of the route between two nodes $(\mathrm{s}, \mathrm{d}), \quad r=1,2, \ldots k, r \in\left\{R^{s, d}\right\} ; h_{r}-$ length of the route; 
$h_{\text {min }}-$ length of the shortest route; $W_{r}^{*}-$ the number of wavelengths available on a route $r$ considering that the nodes of the route may be optical convector $r=1,2, \ldots k, r \in\left\{R^{s, d}\right\} ; W_{\max }-$ maximum number of wavelengths available on all routes.

The basic algorithm has been added to the procedure for selecting a location optical converter. We have investigated two variants of modification, the so-called "narrow" and "broad" methods.

The goal of the "narrow" method is to find a bottleneck in the network (which is set to host a maximum number of connections), where an optical convector is established.

The aim of the "broad" method is to find a place in the network wide area (node, through which minimum number of connections is established), where an optical convector is established.

The consequence is the emergence of FWM signals [13], including those at wavelengths corresponding to the other working channels, what can lead to an increase of errors and poor efficiency of DWDM.

To account the impact of this phenomenon when choosing a route has been used [24], formula for calculating the Q-factor and the associated probability of error may be written as follows:

$$
\begin{aligned}
& Q \approx \frac{P_{6 x}}{\sqrt{P_{\text {ease } \Sigma}+P_{e Y B C \Sigma}}}, \\
& P_{\text {ou }}=\frac{e^{-\frac{Q^{2}}{2}}}{\sqrt{2 \pi} Q} .
\end{aligned}
$$

Expression (7) is used in the calculation of the probability of selecting a route in BCO-RWA algorithm. At the stage of calculating the probability of choosing a route between source and destination, there is a comparison of the calculated Q-factor on each optical channel, through which the route is made with a valid Q-factor value. If the value of Q-factor exceeds the permitted limit, the probability of choosing this route is reduced.

\subsection{Numerical Results and Analysis}

The experimental program was written in JAVA. The program runs on a computer with the following parameters: intel core Duo $2.0 \mathrm{GHz}$, RAM 3Gb. The results of the experiment are shown in the Table 1 , including results without the phenomena of four-wave mixing algorithm. Number of wavelength is $40, \mathrm{k}$ indicated the number of optical converters. Table 1 shows that at small dimensions topologies with 6,7 nodes the "broad" method is better than the "narrow". However, when the dimension of topology allows more connections and more rational arrangement of the convector the "narrow" method. As the number of convectors used in the network increases, rational arrangement of convectors is growing too. Results based on four-wave mixing are presented in the following graphs (Fig. 2).

\begin{tabular}{|c|c|c|c|c|c|c|}
\hline \multicolumn{2}{|c|}{ Nodes 6} & 7 & 8 & 9 & 10 & 11 \\
\hline \multicolumn{7}{|c|}{ Basic method } \\
\hline$k=1$ & 210 & 260 & 295 & 325 & 355 & 390 \\
\hline$k=2$ & 225 & 280 & 315 & 340 & 380 & 410 \\
\hline$k=3$ & 245 & 307 & 335 & 371 & 403 & 448 \\
\hline \multicolumn{7}{|c|}{ Method «wide» } \\
\hline$k=1$ & 221 & 272 & 299 & 328 & 362 & 392 \\
\hline$k=2$ & 234 & 299 & 321 & 343 & 379 & 414 \\
\hline$k=3$ & 263 & 332 & 340 & 372 & 402 & 447 \\
\hline \multicolumn{7}{|c|}{ Method «narrow» } \\
\hline$k=1$ & 211 & 258 & 311 & 341 & 376 & 408 \\
\hline$k=2$ & 224 & 282 & 336 & 365 & 410 & 438 \\
\hline$k=3$ & 246 & 308 & 365 & 402 & 440 & 488 \\
\hline
\end{tabular}

Table 1. Result of the reseach.

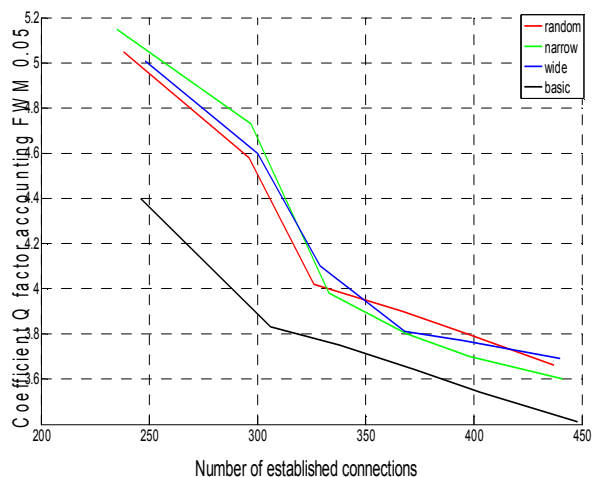

a)

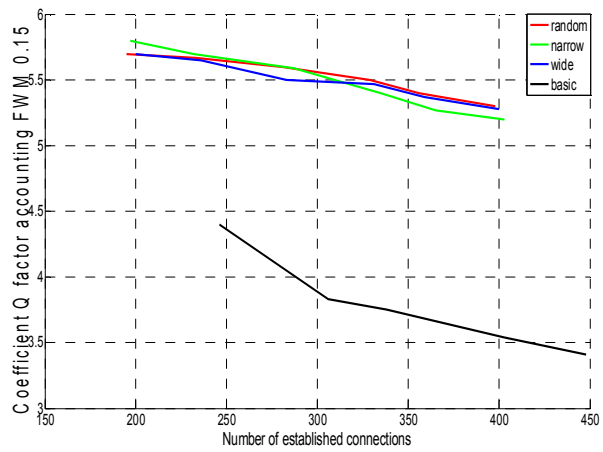

b) 


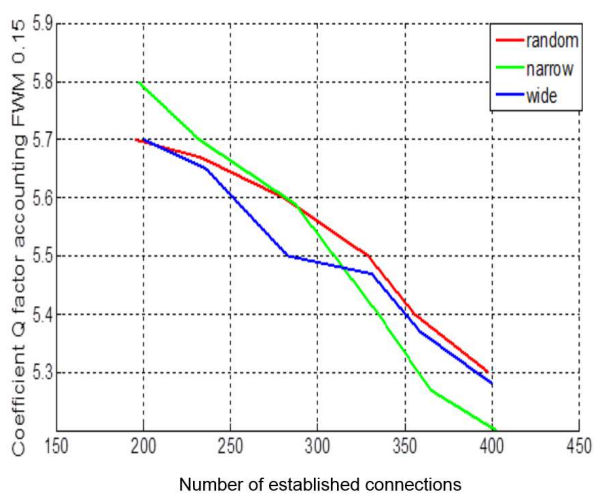

c)

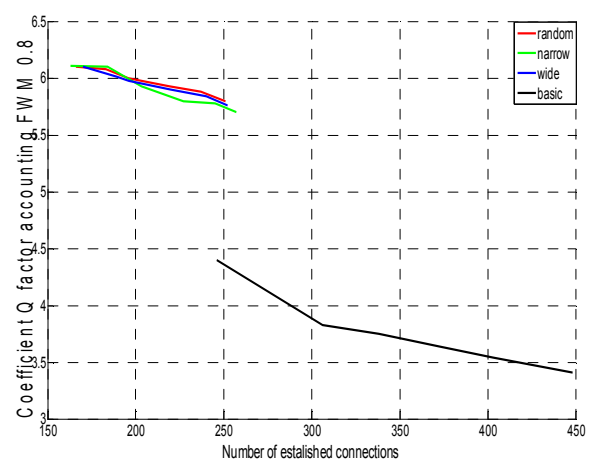

d)

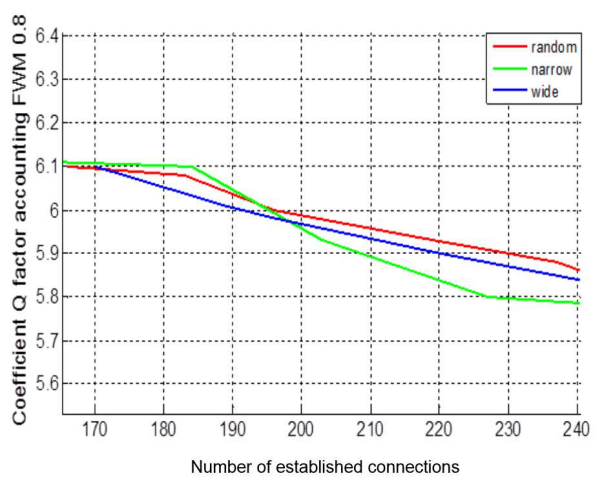

e)

Figure 2.Dependence of $Q$-factor of the number of established connections with different values on the FWM coefficient and network dimension.) Coefficient of a) FWM 0.05, b) FWM 0.15, d) FWM 0.8; c) enlarged part of fig. b); e) enlarged part of fig. $d$ ).

Fig. 2 shows that with increasing FWM ratio (ratio shows values, decreasing in the probability of route selection algorithm, if the Q-factor is less than the normalized value) number of established connections is reduced, since the priority is given quality assurance. As the number of established connections decreases, Q-factor increases due to fewer amount of channels involved in the optical wavelength.

From the analysis of the obtained results we can make a conclusion that, even with a small FWM value $(0.05)$ the value of Q-factor is much higher, though the number of established connections are slightly different at the base method of BCO-RWA algorithm.

\section{Conclusion}

Solving the design problem of RoF technology is a very complicated process, because it is necessary to design two technologies. That is why this paper provides a new method of designing RoF, which is based on the principle of decomposition, thus, there are three main sub-tasks. Major subtasks are distinguished:

Optimal topology for spatial planning antenna synthesis, solution RWA. method of solving spatial planning antennas allows subscriber density, interference noise, power and signal attenuation, the radius of the antenna cover.

After solving the problem of territorial planning of antennas, the data (coordinates of the antennas) should be used as input data to solve the problem of synthesis of the network topology.

To solve the problem of synthesis of network topology a modified method of M-structure should be used, which allows the synthesis of a topology for any connectivity, and considers the case when it is necessary to use the existing topology.

Using the Yen's algorithm for search a set of k-shortest paths. The input is a set of shortest routes for a set of nodes and channels of communication network.

But to solve the problems of RWA for a large WDM network, topology should use heuristic methods for the solution .One of the representatives of the heuristic method for solving the RWA is algorithm BCO-RWA.

However, this method has weakness: it does not solve the problem of choosing the location of the convector of the optical network (set to the first node route without the possibility of installing the connection) during the calculation of the probability of selecting a route it does not consider convectors in nodes through which the route is made in the algorithm; it is limited to only one convector; it does not include the impact of four-wave mixing in the installation of light paths.

To remove these shortcomings several modifications were made: introduction of algorithms for choosing the location, optical convector, modification of the formula for calculating the probability of selecting a light route taking into account the fact that the node routes may have an optical convector, adding mathematical formulas to FWM to calculate the probability of selecting a light route.

The experiment on the software which generates the network topology and solves the problem with RWA algorithm BCO-RWA was made. The results of the analysis obtained in the broad method are effective for small dimensions $(6,7)$ networks and the narrow method is better on a high dimension network topologies, the average gain of 5\% in a single optical convector, with two convectors can gain $7 \%$, with three convectors - $9 \%$.

This approach is recommended for use in the design of optical networking technology based DWDM. The broad method is widely used for small dimensions of network 
topologies (no more than 7 nodes), the narrow method is used for the large dimensions of the network (more than 8 nodes in the network).

\section{Reference}

[1] P. H. Gomes, N.L.S. da Fonseca,O.C. Branquinho "Optimization of the use of Radio Resource of Radio-Over-Fiber Access Networks," Global Telecommunications Conference (GLOBECOM 2010), pp.1-5, Dec. 2010.

[2] A.M.J. Koonen, M. GarcíaLarrodé, "Perspectives of Radio over Fiber Technologies," Journal of Optical Fiber Communication (OSA), 2008.

[3] Jianjun Yu et al, "Radio-over-optical-fiber networks: introduction to the feature issue", Journal of Optical Networking, Vol. 8(5) ,pp 488-481, 2009.

[4] MirosławKlinkowski, MarekJaworski, DavideCareglio "Channel Allocation in Dense Wavelength Division Multiplexing Radio-over-Fiber Networks", in 12th International Conference on Transparent Optical Networks, Munich, Germany, Jun. 2010, pp. 1-5.

[5] Lee, K., Park, S., Choe, K.-I. and Park, C. "Routing and wavelength assignment in WDM all-optical networks." IEEE Electron. Lett., 36, 2000, pp. 964-966.

[6] R.S. Barpanda, A.K. Turuk, B. Sahoo, and B. Majhi, "Genetic Algorithm Approaches to Solve RWA Problem in WDM Optical Networks", ;in Proc. SEMCCO, 2010, pp.599-606.

[7] P. Grønsund, P.E. Engelstad, T. Johnsen, and T. Skeie, "The physical performance and path loss in a fixed WiMAX deployment", Proceedings of the 2007 International Conference on Wireless Communications and Mobile Computing, Honolulu, HI, August 2007.

[8] A. Gamst, "Homogeneous distribution of frequencies in a regular hexagonal cell system", IEEE Transactions on Vehicular Technology, 31(3), 1982, pp. 132-144.

[9] Mokhtar, A., Azizoglu, M. "Dynamic route selection and wavelength assignment in all-optical networks." In IEEE Lasers and Electro-Optics Society Annual Meeting, San Francisco, CA, USA, Oct. , 1995, pp. 220-221.

[10] Yan Zhang "WiMAX Network Planning and Optimization." Auerbach Publications, pp. 372-376, 2009.

[11] Soriano P., Wynants C., Seguin R., Labbe M., Gendreau M., Fortz B. "Design and dimensioning of survivable SDH SONET networks" Telecommunications Network Planning, Kluwer Academic, pp. 148-167, 1999.

[12] Gannett J.W. Fast efficient equipment placement heuristics for broadband switched or internet router networks // TelcordiaTechnologies., pp. 3-19, 1998.
[13] Jin Y. Yen "Finding the K Shortest Loopless Paths in a Network", Management Science, Vol. 17, No. 11, Theory Series, pp. 712-716, 1971

[14] D. Banerjee and B. "Mukherjee, Wavelength Routed Optical Networks Linear formulation resource budgeting tradeoff and a reconfiguration study" in IEEE/ACM Transactions on Networking,vol. 8, № 5, pp. 684-696, 2000.

[15] D.V. Ageyev, V.K. Kovalchuk, A.A. Pereverzev "Planirovanieraspredeleniyadlinvolnpriproektirovaniitransprortnoyseti DWDM [Planning for the distribution of wavelengths in the design of DWDM transport network]", Eastern European Journal of Enterprise Technolopgies, No 5/3(53), pp. 25-29, 2011.

[16] Dmitry Ageyev, Alexander Pereverzev "Wavelength Assignment in Design DWDM Transport Network Using Algorithm BCO-RWA",.in Proceedings of the XIth International Conference on Modern Problems of Radio Engineering, Telecommunications, and Computer Science, TCSET'2012, pp. 277-278, 2012.

[17] M. Sridharan, M.V. Salapaka and A.K. Somani, "A practical approach to operating survivable WDM networks", IEEE Journal on Selected Areasof Communications, vol. 20, № 1. pp. 34-36, 2002.

[18] G.D. Morley and W.D.Grover, "Tabu search optimisation of optical ring transport network," in proc. of IEEE Globecom’01. San Antonio, vol. 4, № 1, pp. 2160-2164, 2001.

[19] S.Yan, M.Ali and J.Deogun, "Route optimisation of multicast sessions in sparse light-splitting optical networks," in Proc. of IEEE Globecom 01, vol. 4, № 1, pp. 2134-2138, 2001.

[20] B. Mukherjee, D. Banerjee and A. Mukherjee, "Some Principles ofdesigning a wide-area WDM optical network" IEEE/ACM Transactions on Networking, vol. 5, № 5, pp. 489-500, 1995.

[21] Rodriguez-Dagnino R.M. and Lopez-Cuadana E.O., "Simulated Annealing and Stochastic ruler algorithms for wavelength assignment planning in WDM optical networks," in Poc. of IEEE SMC'99, vol. 6, pp. 1015-1020, 1999.

[22] M. Ali, B.Ramamurthy and J.S.Deogun, "Routing algorithms for all-optical networks with power consideration : The unicast case," in Proc of the 8th IEEE ICCCN 99. Boston-Natick MA, pp. 335-340, 1999.

[23] D. Teodorović, "Bee Colony Optimization (BCO)," in Swarm Intelligence for Knowledge-Based Systems, Springer-Verlag, Berlin Heidelberg, pp. 39-60, 2009.

[24] K. Inoue "A simple expression for optical FDM network scale considering fiberfour-wave mixing and optical amplifier noise.” Journal of Lightwave Technology, vol. 2, № 5, 1995, pp. 856-861. 